\title{
Stress-Assisted Shock-Wave-Induced Thin-Film Delamination (SWIFD) of CIGS on a Flexible Substrate
}

\author{
Pierre Lorenz*, Igor Zagoranskiy*, Joachim Zajadacz*, Martin Ehrhardt**, Bing Han**, Lukas Bayer*, Klaus Zimmer* \\ ${ }^{*}$ Leibniz Institute of Surface Modification, Permoserstr. 15, 04318 Leipzig, Germany \\ ** Laser-Material Interaction Lab, 2011 Co-innovation Center, Nanjing University of Science \& \\ Technology, 210094 Nanjing, People's Republic of China \\ E-mail: Martin.ehrhardt@njust.edu.cn
}

\begin{abstract}
The structuring of thermal-sensitive functional layers by laser patterning methods remains a challenge. The shock-wave-induced thin-film delamination (SWIFD) process is a non-thermal process of mechanically-induced film removal where the process exhibits an extraordinary application potential. Due to the spatial separation of laser ablation that is performed at the rear side of the substrate and the shock-wave-induced thin film delamination at the substrate's film side the thermal impact to the thin films is excluded. The SWIFD process for CIGS solar cells on polyimide substrates was studied for dependence on externally applied tension forces F. The resulting SWIFD patterns are imaged by scanning electron microscopy (SEM) to analyze the geometrical shape and the surface morphology. A reduction of the CIGS delamination threshold fluence $\Phi_{\text {th }}$ as well as a slight increase of the delaminated area A with increasing tension forces $\mathrm{F}$ is found. Like previous results, the area $\mathrm{A}$ increases with growing laser fluence and number of laser pulses and the $\Phi_{\text {th }}$ decreases with increasing number of laser pulses. The non-thermal nature of the SWIFD process, enabling a film-selective delamination of the CIGS layer (including the front contact) without damaging the Mo back contact, is also shown.
\end{abstract}

DOI: 10.2961/jlmn.2017.02.0008

Keywords: SWIFD, delamination, nanosecond laser, CIGS, solar cell, shock wave

\section{Introduction}

The structuring of thermal sensitive functional layers is of high importance for the further development of flexible electronic devises. Especial patterning of thin film solar cell such as CIGS (copper indium gallium selenide) for edge isolation or serial interconnection for CIGS-module fabrication are of great interested. Mechanical scribing of CIGS is limited in terms of scribing speed, flexibility and distance between two scribes. To overcome these problems laser scribing of CIGS have been investigated extensive [14]. However laser patterning of CIGS or other thin films of com-pound semiconductors is still a challenge as it needs to avoid alteration of functional material properties. Such laser-induced material alterations that often accompany laser ablation processes can be already found at ultrashort laser pulse irradiation. This can result in thermal modification of the scribe edges that influences the functionality of the devices $[\mathbf{1}, \mathbf{5}]$. The usage of alternative methods like the shock-wave-induced thin-film delamination (SWIFD) allows the non-thermal structuring of functional layers due to the spatial separation of the laser ablation process from the delamination process [6-9]. At the SWIFD process, the back side of the substrate - front-side-covered with a functional layer - is irradiated and the following laser ablation process induces a transverse shock wave through the substrate and finally results in a delamination of the functional layer due to the interaction of the shock wave with the functional layer-substrate interface. The SWIFD process was studied at different layer/substrate combinations. As functional layers' CIGS solar cell, indium tin oxide (ITO), and epoxy-based negative photoresist (SU-8) and as flexible substrates polymers (polyimide (PI) and polyethylene terephthalate (PET)), and stainless steel were studied [7-9]. Furthermore, the dynamics of the SWIFD process was studied by shadowgraphy measurements $[8,9]$. The delamination processes of thin films can be discussed in relation to thermal-induced stress effects [10]. The delamination process induced by SWIFD is most likely dominated by the shock wave/interface interaction [9]. For example, the laser-induced shock-wave-generation technique uses the shock wave/interface interaction and can be applied for determination of the adhesion of thin films [11]. In the present study, the SWIFD process on externally stretched CIGS solar cells on polyimide was studied. The aim of this study is to investigate how the process window for the SWIFD process can be extended to reduce the required partial laser ablation of the substrate material.

\section{Experimental set-up}

The stress-assisted shock wave delamination of $\mathrm{CuIn}_{\mathrm{x}} \mathrm{Ga}_{1-\mathrm{x}} \mathrm{Se}_{2}$ (CIGS) material was studied at samples similar shaped as applied for tensile tests. Therefore, the CIGS solar cell material (front contact: indium tin oxide (ITO) with a thickness of $\approx 0.2 \mu \mathrm{m}$, active material: copper indium gallium diselenide (CIGS) $\approx 2 \mu \mathrm{m}$, back contact: molybdenum $(\mathrm{Mo}) \approx 1 \mu \mathrm{m}$, flexible substrate: polyimide (PI) $\approx 25 \mu \mathrm{m}$ (see Fig. 1 (a))) was laser-cut in a bone like shape. An illustration of the laser-cut test specimen is shown in Fig. 1 (b). Samples with a gauge length of $\mathrm{l}=10 \mathrm{~mm}$ and 
different widths $\mathrm{w}$ of $1 \mathrm{~mm}$ and $2 \mathrm{~mm}$ were produced. The CIGS test samples were fixed by means of the clamps (via screws) in a miniaturized force-displacement tester. The principal set-up for SWIFD is sketched in Fig. 1(c). The force to the sample specimen is provided by a linear precision stage (M505.2DG, Physik Instrumente $\mathrm{GmbH}$ ) that is computer-controlled (C-862 mercury, Physik Instrumente $\mathrm{GmbH}$ ). The stage can move in steps of $0.05 \mu \mathrm{m}$ and provides a constant velocity of $25 \mu \mathrm{m} / \mathrm{min}$. The actual force at the samples is measured by a force sensor (KD24S, $\pm 50 \mathrm{~N}$ with a full range of $\pm 40 \mathrm{~N}, \mathrm{ME}$ Meßsysteme $\mathrm{GmbH})$. A strain gage with measuring amplifier (GSV_3, ME Meßsysteme $\mathrm{GmbH}$ ) allows a measurement accuracy of $0.1 \%$ with output signals as low as $0.5 \mathrm{mV} / \mathrm{V}$. The whole set-up is computer-controlled. Force and displacement are automatically recorded during the experiment.

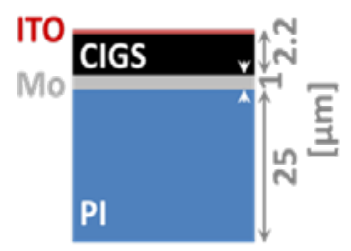

(a)

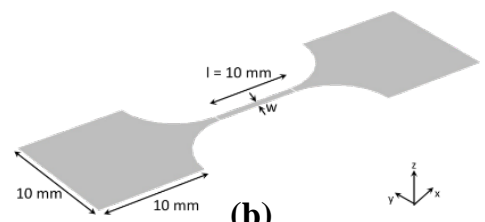

(b)

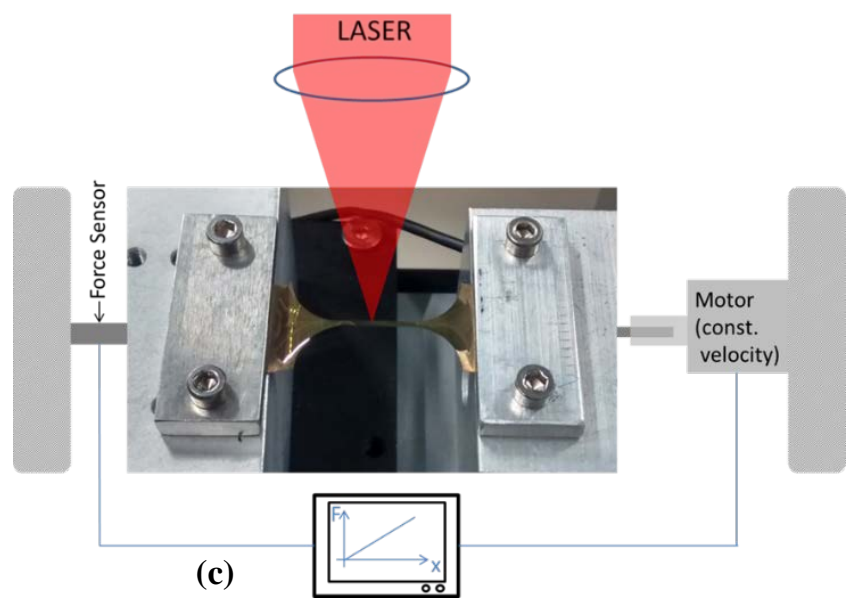

Fig. 1 (a) Schematic of the cross section of the used CIGS solar cell material (front contact: indium tin oxide (ITO), active material: copper indium gallium diselenide (CIGS), back contact: molybdenum (Mo), flexible substrate: polyimide (PI)). (b) Geometry of the laser-cut sample with a gauge length $\mathrm{l}=10 \mathrm{~mm}$ and gauge width $\mathrm{w}$ (The sketch shows a structure with $\mathrm{w}=1 \mathrm{~mm}$ ). (c) Schematic of the experimental set-up.

A typical force-displacement curve of a $25 \mu \mathrm{m}$ thick polyimide substrate measured with the described set-up is shown in Fig. 2 (a). The analysis of the measurement allows the estimation of the Young's modulus of (7.58 \pm 0.01$) \mathrm{GPa}$ as well as of the ultimate tensile stress of $\sigma_{\mathrm{u}} \approx 90 \mathrm{MPa}$ at a maximal elongation of $\approx 5 \%$. The stretch of the CIGS solar cell on polyimide presents an almost similar behaviour, see Fig. 2 (b). However, at $F_{\mathrm{n}}=1.48 \mathrm{~N} / \mathrm{mm}, 1.69 \mathrm{~N} / \mathrm{mm}$, and $1.86 \mathrm{~N} / \mathrm{mm}$ additional features can be detected. The additional features can be explained by a cracking of the CIGS solar cell thin films including the molybdenum back contact exposing the polyimide substrate. For the first feature at a normalized tension force of $1.48 \mathrm{~N} / \mathrm{mm}$ at an elongation of $1.06 \%$ a stress inside the Mo back contact of $\approx 860 \mathrm{MPa}$ can be estimated using a linear elastic model:

$$
\vec{\nabla} \cdot \vec{\sigma}=\vec{F}
$$

with $\vec{\sigma}$ Cauchy stress tensor.

This value is in the order of the ultimate tensile stress for molybdenum of roughly 0.485 to $0.69 \mathrm{GPa}$ [12]. To avoid CIGS thin-film cracking observed in these preliminary force-displacement measurements before the shock wave delamination experiments the applied normalized tension forces were limited to $F_{n}<1 \mathrm{~N} / \mathrm{mm}$. The PI irradiationinduced shock wave pressure can be estimated by Phipps et al. $[8,13]$ :

$$
p(\Phi) \approx 37.8 \frac{M P a \cdot c m^{1.4}}{J^{0.7}} \cdot \Phi^{0.7}
$$
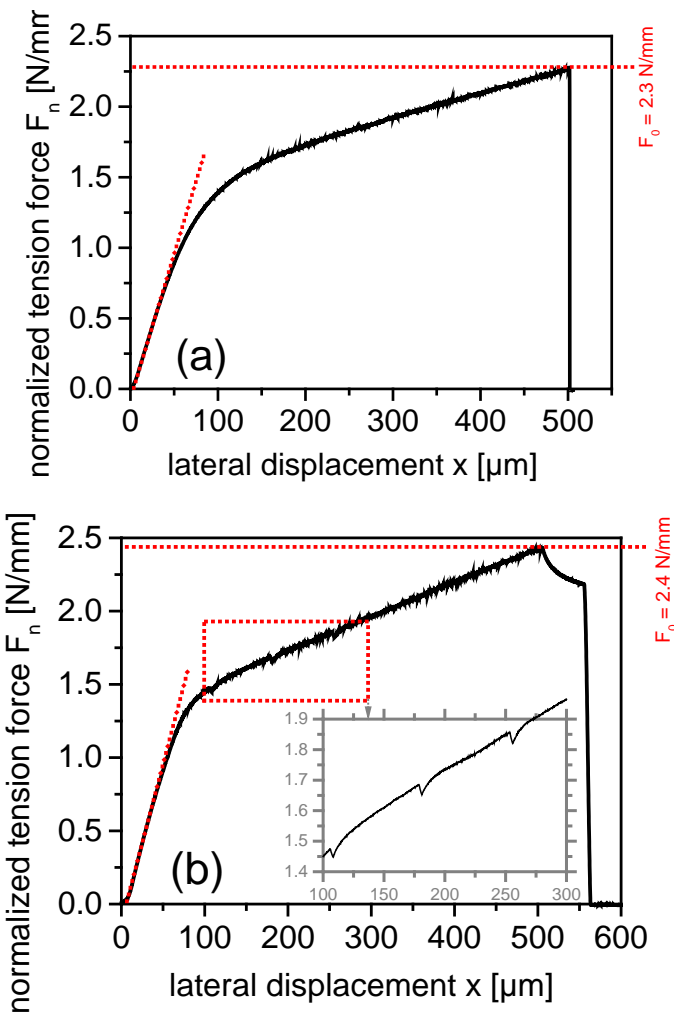

Fig. 2 Normalized tension force $F_{n}\left(F_{n}=F / w, w=1 m m\right.$ gauge width) in dependent on the lateral displacement $x$ of: (a) a stretched PI substrate (thickness $25 \mu \mathrm{m}$ ), (b) $\mathrm{F}_{\mathrm{n}}(\mathrm{x})$ at a stretched CIGS solar cell (ITO/CIGS/Mo) on a polyimide substrate

Within the shock-wave-delamination process studies the rear side of the polyimide substrate of the stretched sample was irradiated with a $\mathrm{KrF}$ excimer (Exitech, Ltd ) having a wavelength of $\lambda=248 \mathrm{~nm}$ and a pulse duration of $t_{p}=25 \mathrm{~ns}$. The homogenized laser beam has been projected to a spot size of $100 \mu \mathrm{m} \times 100 \mu \mathrm{m}$ with an overall energy deviation of below $5 \%$. In addition to the tensile force the number of laser pulses $\mathrm{N}$ and laser fluences $\Phi$ were varied. An overview of the interaction processed between the UV laser pulses and the polyimide flexible substrate as well as the surface morphology of the laser irradiated polyimide is given in ref. $[14,15]$. The ablation rate $\Theta$ of the polyimide is in the fluence range $\Phi=1.6-2.9 \mathrm{~J} / \mathrm{cm}^{2}$ used between 
$\Theta=320 \mathrm{~nm} /$ pulse and $\Theta=430 \mathrm{~nm} /$ pulse $[14,16]$. Therefore with highest laser pulse number $\mathrm{N}=10$ and the highest laser fluence $\Phi=2.9 \mathrm{~J} / \mathrm{cm}^{2}$ used in the present investigation the min. remain thickness of the polyimide substrate is $>20 \mu \mathrm{m}$ which ensure the stability of the polyimide substrate and the functional CIGS layer. In ref. [17] a damping coefficient in polyimide of $\alpha \sim 0.032 \mu \mathrm{m}(1 / \alpha \sim 32 \mu \mathrm{m})$ for a shock wave generated by a UV nanosecond laser pulse $\left(\lambda=248 \mathrm{~nm}, \mathrm{t}_{\mathrm{p}}=25 \mathrm{~ns}\right)$ have been reported. Therefore the penetration depth of the shock wave is larger than the thickness of the polyimide substrate which is essential for the SWIFD process. The resulting structures of the SWIFD processed areas were analysed by scanning electron microscopy (SEM). In Fig. 3 a sketch of the processes involved during the SWIFD process is shown.
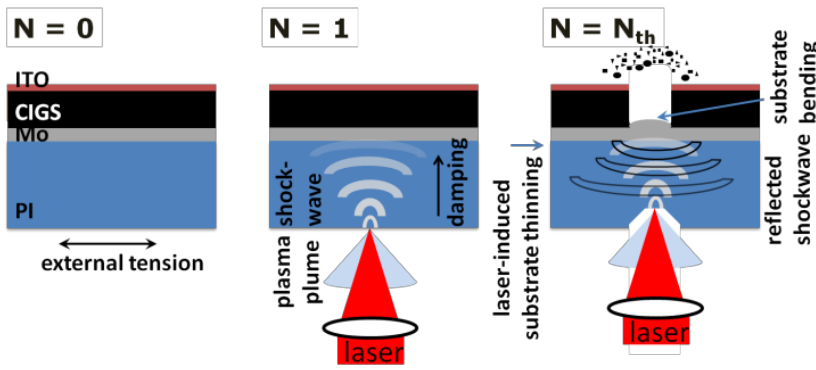

Fig. 3 Sketch of the SWIFD process. Initalisation: $(\mathrm{N}=0)$ The sample is loaded with the external force. After applying a laser pulse $(\mathrm{N}=1)$, a Shock wave is generated inside the polyimide substrate. The shock wave and the bending of the polyimide substrate causes a cracking and delaminating of the functional layers. In some cases $\left(\mathrm{N}=\mathrm{N}_{\mathrm{th}}\right)$, multiple pulse irradiation of the polyimide substrate are required before a delaminating can be achieved.

\section{Results}

At first, the appearance of CIGS thin-film delamination due to the rear side irradiation of the polyimide was studied. In Fig. 4, the SWIFD effect on the sample's films are summarized for different laser parameters with $\left(F_{n}=0.1-\right.$ $0.4 \mathrm{~N} / \mathrm{mm})$ and without $\left(F_{n}=0 \mathrm{~N} / \mathrm{mm}\right)$ with external tension force. At suited combinations of laser pulse numbers $\mathrm{N}$ and laser fluences $\Phi$ the irradiation results in a delamination of the CIGS including the ITO front contact, as seen in Fig. 4 green marked. The delaminated areas were analysed by SEM. Characteristic patterns for non-stretched $\left(F_{n}=0 \mathrm{~N} / \mathrm{mm}\right)$ and stretched $\left(F_{n}=0.4 \mathrm{~N} / \mathrm{mm}\right)$ samples that were achieved with three laser pulses at a laser fluence of $2.9 \mathrm{~J} / \mathrm{cm}^{2}$ are shown in Fig. 5. (top).

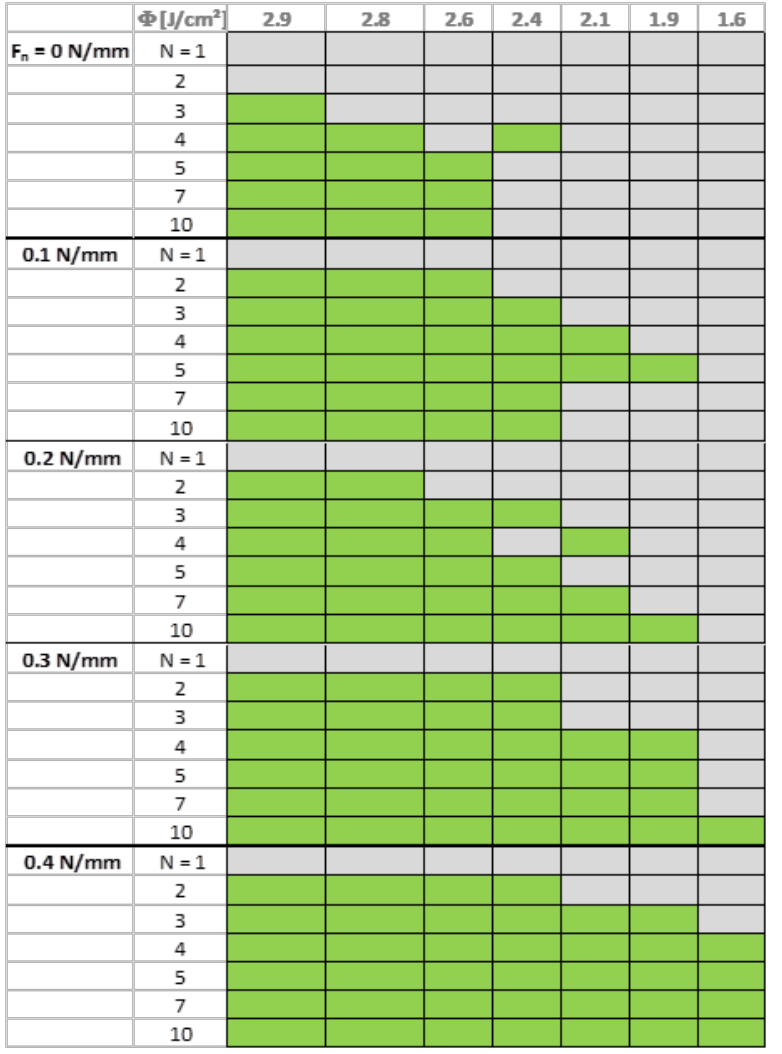

Fig. 4 Summary of the effects of the stress-assisted shock wave delamination of CIGS at different numbers of laser pulses $\mathrm{N}$, laser fluences $\Phi$ and at various tension forces $\mathrm{F}$ (green: delamination of CIGS, grey: no effect) $\Delta \mathrm{F}=0.1 \mathrm{~N}, \Delta \mathrm{X} / \Phi=10 \%$.

At both samples, the irradiation results in a delamination of the CIGS from the Mo back contact with jagged edge that is characteristic of SWIFD. The SEM images neither show thermal modifications of the CIGS edge nor laser irradiation-induced modifications of the exposed Mo surface like cracks. However, the SEM images present that the delaminated area is larger for the stretched samples compared to non-stretched samples. Based on the experimental results (see Fig. 4) the estimation of the CIGS delamination laser fluence threshold $\Phi_{\text {th }}$ is possible. The threshold $\Phi_{\text {th }}$ dependence on the externally normalized force $F_{n}$ for $N=2,3$, and 7 is summarized in Fig. 5 (bottom). In general, the $\Phi_{\text {th }}$ decreases with increasing $F_{n}$ as well as with increasing laser pulse number $\mathrm{N}$. 

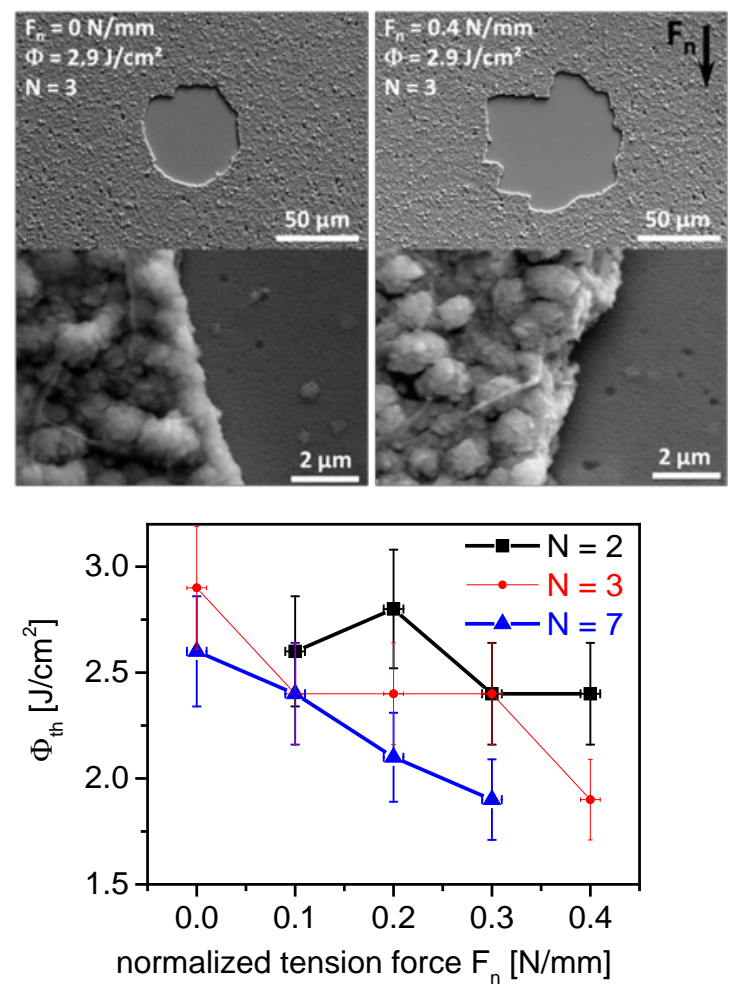

Fig. 5 (top) SEM image of irradiated samples $\left(\Phi=2.9 \mathrm{~J} / \mathrm{cm}^{2}\right.$, $\mathrm{N}=3)$ without external tension force $\left(\mathrm{F}_{\mathrm{n}}=0 \mathrm{~N} / \mathrm{mm}\right)$ and with externally normalized tension force $\left(F_{n}=0.4 \mathrm{~N} / \mathrm{mm}\right)$ (The stretch direction is marked in the image by a black arrow.). (bottom) CIGS delamination threshold $\Phi_{\text {th }}$ dependence on the normalized tension force $\mathrm{F}_{\mathrm{n}}$ at $\mathrm{N}=2,3$, and 7 .

Further, the delaminated areas at different laser fluences (see Fig. 6 (top)) as well as at different numbers of laser pulses (see Fig. 6 (bottom)) were measured. The measurements presented for the used parameters (see Fig. 6 (top) and (bottom)) the size of the delaminated area increases with increasing laser fluence and number of laser pulses. Further, the SEM images show that the presented parameters allow the CIGS delamination without damaging the Mo back contact.

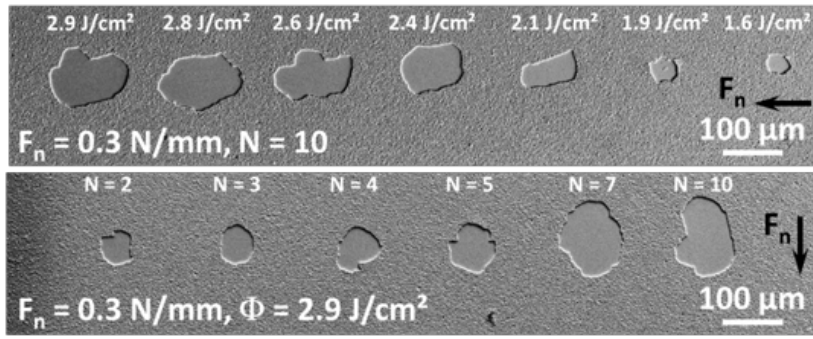

Fig. 6 Exemplary SEM images of the delamination-patterned CIGS solar-film material for different laser-irradiation parameters: (top) fluences $\Phi=1.6-2.9 \mathrm{~J} / \mathrm{cm}^{2}$ at $\mathrm{N}=10$ and $\mathrm{F}_{\mathrm{n}}=0.3 \mathrm{~N} / \mathrm{mm}$, and (bottom) laser pulses $\mathrm{N}=2-10$ at $\Phi=2.9 \mathrm{~J} / \mathrm{cm}^{2}$ and $F_{n}=0.3 \mathrm{~N} / \mathrm{mm}$ (The stretch direction is marked in the image by a black arrow.).

In the SEM images of the delaminated area shown in Fig. 6 it can be seen that for high laser fluencies and laser pulse number the shape of the delaminated area is elongate parallel to the direction of the applied external force. Whereas for low laser fluencies and laser pulse numbers used the shape of the delaminated area is roughly round and therefore independent from the applied external force direction. In Fig. 7 a summary of the ratio between the elongation of the delaminated area along the axis parallel $\mathrm{L}_{\text {parallel }} \mathrm{Fn}$ and perpendicular $\mathrm{L}_{\text {perpendicular } F n}$ to the direction of the external force is shown, respectively.
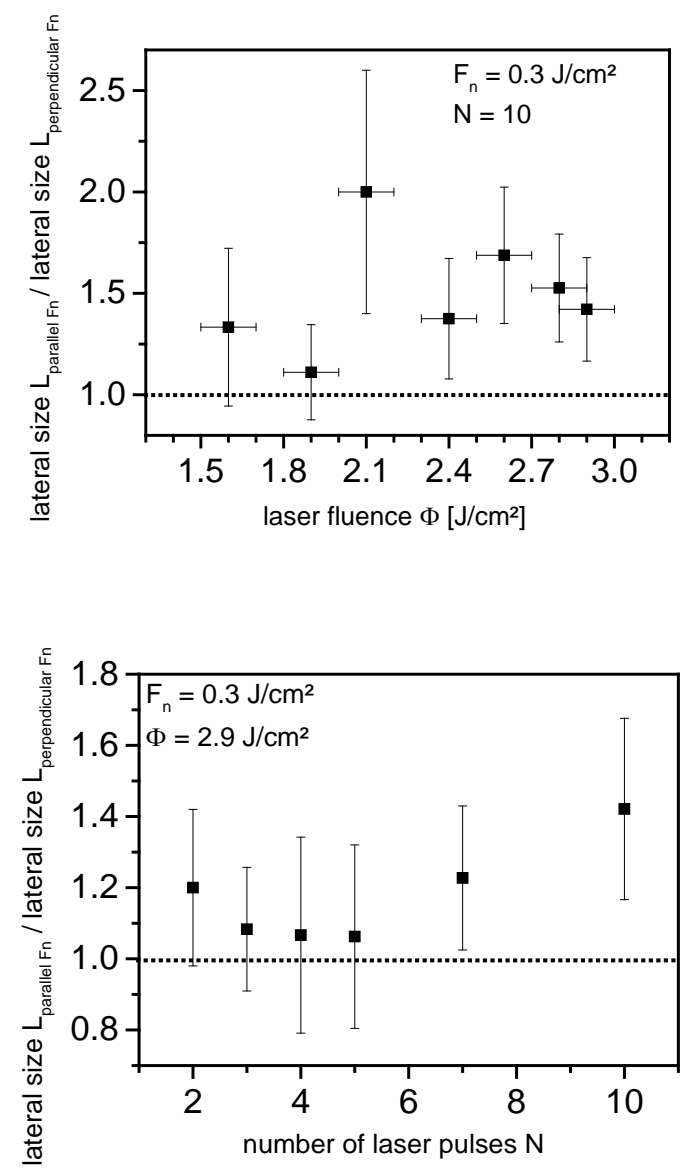

Fig. 7 Ratio between the between the elongation of the delaminated areas shown in Fig. 6 along the axis parallel $\mathrm{L}_{\text {parallel } F n}$ and perpendicular $\mathrm{L}_{\text {perpendicular } F n}$ to the direction of the external force is shown, respectively. (Top) Ratio in dependence on the laser fluence used and (bottom) applied laser pulse number, respectively.

In Fig. 8 (a)-(c) the delaminated area is summarized dependent on the laser fluence, the number of laser pulses, and the normalized tension force, respectively. In agreement with the SEM images at different external tension forces, seen in Fig. 5 (top), a more detailed analysis of the measurements presents that the delaminated CIGS area increases with increasing normalized tension force (Fig. 8 (c)). 

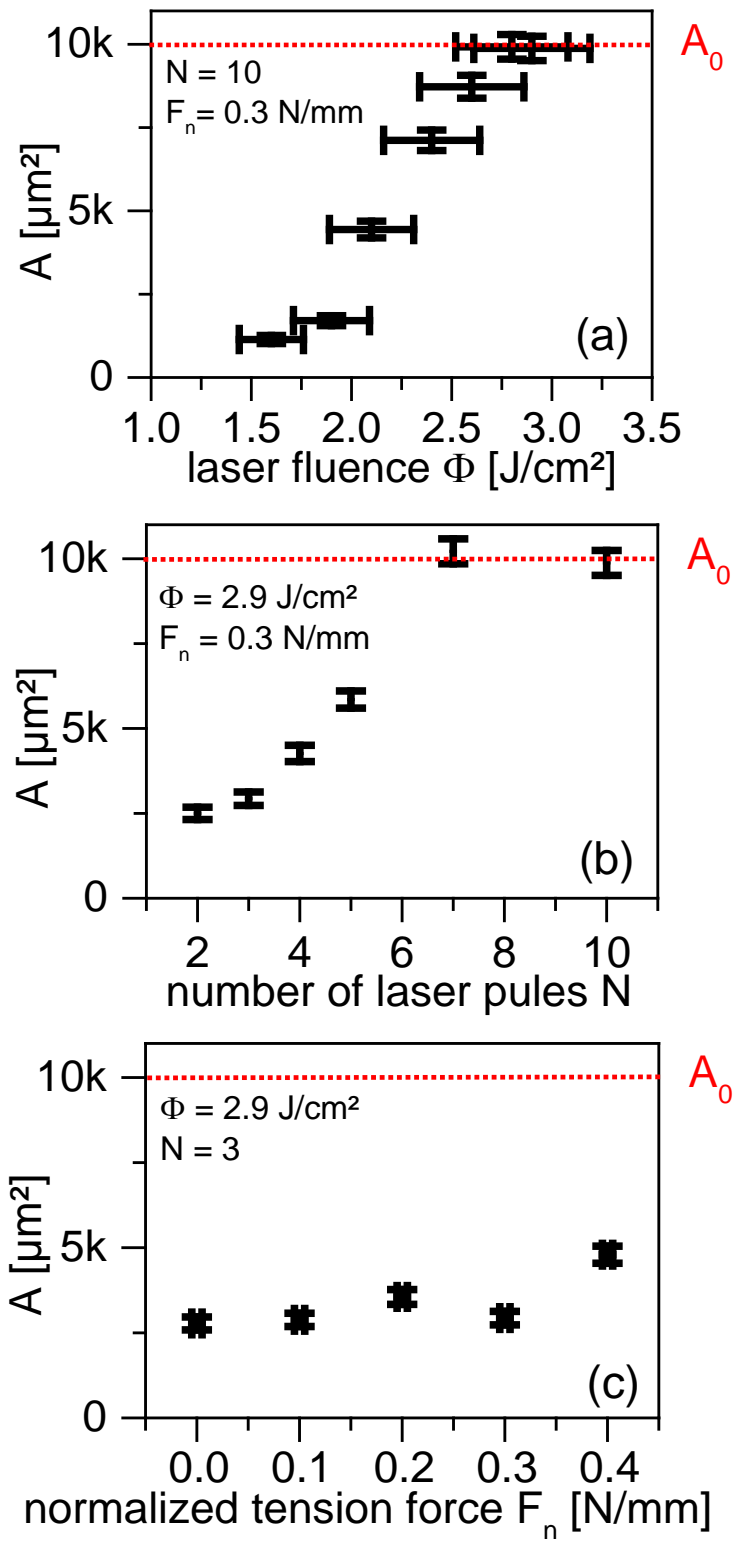

Fig. 8 Dependence of the delaminated CIGS area A on the:

(a) laser fluence $\Phi\left(\mathrm{A}_{0}=100 \times 100 \mu \mathrm{m}^{2}\right.$ laser-irradiated area $)$

(b) number of laser pulses $\mathrm{N}$, and

(c) normalized tension force $\mathrm{F}_{\mathrm{n}}$.

\section{Discussion}

The rear-side irradiation of a PI substrate - front side covered by a CIGS solar cell (ITO: front contact, CIGS: active layer, Mo: back contact) - allows the selective removal of the CIGS including the ITO due to the stress generated by the laser-induced shock wave and the externally applied forces. The CIGS delamination process was studied depending on the laser parameters (laser fluence $\Phi$ and number of laser pulses $\mathrm{N}$ ) as well as depending on an external tension force and externally induced stress, respectively. A sufficient high laser fluence is necessary for the selective delamination of the CIGS where the delamination threshold $\Phi_{\text {th }}$ is dependent on the number of laser pulses $\mathrm{N}$ as well as on the externally normalized tension force $F_{n}$. In general, $\Phi_{\text {th }}$ decreases with increasing $\mathrm{N}$ and $\mathrm{F}_{\mathrm{n}}$ (see Fig. 5 (bottom)). Further, the delaminated CIGS area increases with the laser fluence, number of laser pulses and externally normalized tension force, respectively. The observed effects are summarized in Tab. 1.

Table 1 Summary of the different behaviours: Dependency of the CIGS delamination threshold $\Phi_{\text {th }}$ on the number of laser pulses $\mathrm{N}$ and on the externally normalized tension force $\mathrm{F}_{n}$ and dependency of the delaminated CIGS area A on N, $\mathrm{F}_{\mathrm{n}}$ and on the laser fluence $\Phi$. ( $\uparrow$ : increasing, $\downarrow$ : decreasing, $\nearrow$ : slightly increasing).

\begin{tabular}{|c|c|c|}
\cline { 2 - 3 } \multicolumn{1}{c|}{} & $\begin{array}{c}\text { threshold } \\
\boldsymbol{\Phi}_{\text {th }}\end{array}$ & $\begin{array}{c}\text { delaminated } \\
\text { area } \\
\text { A }\end{array}$ \\
\hline $\begin{array}{c}\text { number of laser pulses } \\
\mathbf{N}\end{array}$ & $\downarrow$ & $\boldsymbol{\top}$ \\
\hline $\begin{array}{c}\text { normalized tension force } \\
\text { F }\end{array}$ & $\downarrow$ & $\nearrow$ \\
\hline $\boldsymbol{\Phi}$ & & $\uparrow$ \\
\hline
\end{tabular}

The physical processes, included in the delamination mechanism are now summarized for the discussion of the obtained results. The irradiation of the polyimide substrate results in a laser ablation of the PI on the rear side of the substrate. Further, the ablation process induces a shock wave which traverses through the substrate. The traverse shock wave is damped in the substrate where a damping coefficient $\alpha$ of $1 / \alpha=32 \mu \mathrm{m}$ can be estimated [17]. Further, a divergence of the shock wave cannot be completely excluded. In addition, the interaction of the shock wave with the layer-substrate interface induces a stress inside the layer system and at sufficient stress a delamination process can be induced.

Two different laser ablation-induced processes must be considered to estimate the stress in the layer system:

(1) The rear side laser irradiation induces a bending of the front side of the substrate including the layer system [8] where the bending induces a stress in the layer system.

(2) Reflections of the shock wave at the layer-substrate interface can result in complex stress fields inside the layer system.

For the CIGS solar cell material on polyimide, the shock wave reflection process is most likely dominating [9]. In the current experiments this laser-induced stress is superimposed by the homogeneous stress within the films. The $\Phi_{\text {th }}$ decreases with increasing $N$ and $F_{n}$. The multipulse irradiation results in a thinning of the substrate and thereby in a higher shock wave pressure on the layersubstrate interface due to the reduced damping and substrate thickness, respectively. The external tension force induces a stress inside the layer system e.g. at $F_{n}=0.3 \mathrm{~N} / \mathrm{mm}$ in the CIGS layer a von-Mises stress of $\sigma_{\mathrm{vM}}=33 \mathrm{MPa}$ can be estimated, where the additional stress reduces the $\Phi_{\text {th. }}$. For example, at $\mathrm{N}=7$ and $\mathrm{F}_{\mathrm{n}}=0 \mathrm{~N} / \mathrm{mm}$ a $\Phi_{\text {th }}$ of $\approx 2.6 \mathrm{~J} / \mathrm{cm}^{2}$ and at $\mathrm{F}_{\mathrm{n}}=0.3 \mathrm{~N} / \mathrm{mm}$ a $\Phi_{\text {th }}$ of $1.9 \mathrm{~J} / \mathrm{cm}^{2}$ can be found (see Fig. 4). That means, under the estimation of Phipps et al. (see Eq. 2), a reduction of the necessary stress to induce a successful CIGS delamination by 
$\approx 15 \mathrm{MPa}$ is required. The stress which was induced by an external tension force is thereby in the same order of magnitude as the reduction of the shock-wave-induced stress. The rear-side irradiations of a pure polyimide substrate with sufficient laser fluences can result in a plastic deformation of the front-side surface where the maximum deformation can be detected at the center of the irradiated area [16]. The delamination process starts at the center of the irradiated area (see Fig. 6) at low numbers of laser pulses and laser fluences, too. However, based on the geometry (irradiated area $\mathrm{A}_{0}=100 \times 100 \mathrm{~mm}^{2}$, substrate thickness: $25 \mu \mathrm{m})$ and the published shadowgraphy measurements [8] a planar shock wave inside the substrate is most likely and thereby an almost homogenous stress induced by the shock wave reflection is expected.

The reason for the center-localized delamination can most likely be explained by a deformation of the front side of the substrate, e.g. this effect was already detected by shadowgraphy [8] where the maximum deformation can be detected in the center [16]. This most likely slight deformation induces an additional and time-dependent stress distribution in the layer system with a maximum at the center of the irradiation. This effect finally results in the center-localized delamination at low laser fluences whereas at higher fluences the influence of the deformation is reduced and the delaminated area tends to the irradiated area $\mathrm{A}_{0}$.

A further measurement of the time-dependent deformation, e.g. by time-dependent white light interferometry, will clarify this aspect. Further, the increasing of the area with increased normalized tension force $F_{n}$ and number of laser pulses can be explained by the reduction of $\Phi_{\text {th }}$ and the reduced damping, respectively.

\section{Conclusion and Outlook}

The shock-wave-induced thin-film delamination (SWIFD) process on the CIGS solar cell (ITO: indium tin oxide front contact, CIGS: $\mathrm{CuIn}_{\mathrm{x}} \mathrm{Ga}_{1-\mathrm{x}} \mathrm{Se}_{2}$ active layer, Mo: molybdenum back contact) on polyimide substrates was studied dependent on an externally impressed tension force $F_{n}$. The SWIFD process allows the well-defined nonthermal selective delamination of the CIGS layer (including the front contact) without damaging the Mo back contact. The CIGS delamination threshold $\Phi_{\text {th }}$ can be reduced by an additional tension force. Further, the $\Phi_{\text {th }}$ decreases with increasing number of laser fluences. Also, the delaminated CIGS area A can be adjusted by the additional tension force, the area A slightly increases with increasing normalized tension force $F_{n}$. Further, the area $A$ increases with increasing laser fluence and number of laser pulses. The usage of a statically induced additional stress in the film allows the influence of the delamination process. In the next step, the dynamically modulated stress distribution in the layer system by additional laser-induced shock waves and ultrasound seems expedient, respectively. This concept exhibits an outstanding potential for the successful influence of the SWIFD process to improve the delamination process.

\section{Acknowledgements}

This work was supported by the "National Natural Science Foundation of China (NSFC) (No.11402120)", the "Natural Science Foundation of Jiangsu Province (Jiangsu Natural Science Foundation) (No. BK20140796)”, and the "Fundamental Research Funds for the Central Universities (No.30915015104)". We are grateful for funding from the German Research Foundation (DFG: Deutsche Forschungsgemeinschaft) under ZI 660/12-1 and the European Community's Seventh Framework Programme (FP7/2007 2013) under APPOLO project (grant agreement No. 609355).

\section{References}

[1] P. Gecys, R. Gediminas, E. Miltenis, A. Braun, and S Ragnow: J. Laser Micro/Nanoeng., 7, (2012) 33.

[2] S.C. Jeoung, H.-S. Lee, J.S. Yahng, H.K. Lee, H.Y. Moon, K.J. Kim, D.G. Lee, D.H. Park, Y.S. Yu, and S.-J. Ji: Opt. Express, 19, (2011) 16730.

[3] J. Hermann, M. Benfarah, G. Coustillier, S. Bruneau, E. Axente, J.F. Guillemoles, M. Sentis, P. Alloncle, T. Itina: Appl. Surf. Sci., 252, (2006) 4814.

[4] A. Burn, M. Muralt, S. Pilz, V. Romano, R. Witte, B. Frei, S. Buecheler, S. Nishiwaki, and L. Krainer: Physics Procedia, 41, (2013) 713.

[5]A. Wehrmann, S. Puttnins, L. Hartmann, M. Ehrhardt, P. Lorenz, K. Zimmer: Opt. Laser Technol., 44, (2012) 1753.

[6] M. Ehrhardt, A. Wehrmann, P. Lorenz, and K. Zimmer: Appl. Phys. A, 113, (2013) 309.

[7] P. Lorenz, M. Ehrhardt, and K. Zimmer: 8th Int. Conf. on Laser Assisted Net Shape Eng., Furth, (2014) p.1015.

[8] P. Lorenz, T. Smausz, T. Csizmadia, M. Ehrhardt, K. Zimmer, and B. Hopp: Appl. Surf. Sci., 336, (2015) 43.

[9] M. Ehrhardt, P. Lorenz, L. Bayer, C. Molpeceres, C. Ramirez, and K. Zimmer: Proc. SPIE, 9735, (2016) 973503.

[10] J. Bovatsek, A. Tamhankar, R. Patel, N. Bulgakova, and J. Bonse: Thin Solid Films, 518, (2010) 2897.

[11] J.L. Wang, R.L. Weaver, and N.R. Sottos: Exp. Mech., 42, (2002) 74.

[12] Technical Information-Molybdenum, June 2016 http://www.goodfellow.com/E/Molybdenum.html

[13] C.R. Phipps, T.P. Turner, R.F. Harrison, G.W. York, W.Z. Osborne, G.K. Anderson, X.F. Corlis, L.C. Haynes, H.S. Steele, K.C. Spicochi, and T.R. King: Appl. Phys. A, 64, (1988) 1083.

[14] K. Zimmer, R. Böhme, T. Stephan, C. Scheit, and A. Braun: Appl. Surf. Sci., 255, (2009) 9869.

[15] D. Bäuerle: "Laser Processing and Chemistry" (Springer, Berlin Heidelberg, 2011).

[16] P. Lorenz, L. Bayer, M. Ehrhardt, K. Zimmer, and L. Engisch: Proc. SPIE, 9351, (2015) 935119.

[17] P. Lorenz, M. Ehrhardt, L. Bayer, and K. Zimmer: Physics Procedia, 83, (2016) 240.

(Received: February 24, 2017, Accepted: July 30, 2017) 